\title{
Violencia en consumidores de drogas ilícitas reclutados en centros específicos de tratamiento
}

\section{Violence among illicit drug users recruited in drug treatment facilities}

\author{
Elisabet Arribas-Ibar*, Josep Maria Suelves**, Albert Sánchez-Niubò***, \\ Judit Tirado-Muñoz****, Antònia Domingo-Salvany*, Ma Teresa Brugal*****. \\ * Grupo de Investigación en Epidemiología de las Drogas de Abuso. Instituto Hospital del Mar de Investigaciones Médicas \\ (IMIM) de Barcelona. ** Agencia de Salud Pública de Cataluña. *** Unidad de Investigación y Desarrollo, Parc Sanitari Sant \\ Joan de Déu, Fundació Sant Joan de Déu. CIBER en Epidemiología y Salud Pública, CIBERESP, España. **** Instituto Hospital \\ del Mar de Investigaciones Médicas (IMIM) de Barcelona. ***** Agencia de Salud Pública de Barcelona.
}

\section{Resumen}

El uso ilícito de drogas se ha asociado a lesiones producidas por violencia. Nuestro objetivo es estimar en usuarios de drogas ilícitas, la prevalencia de violencia en los últimos 12 meses y estudiar la superposición víctimaagresor. Se reclutaron personas consumidoras de drogas ilícitas (502) en centros de tratamiento de drogas. La violencia se evaluó mediante cuatro preguntas sobre victimización y una sobre agresión referidas a los últimos 12 meses. Las asociaciones entre violencia y aspectos sociodemográficos, consumo de sustancias, delincuencia y mercado de drogas ilegales se analizaron con modelos de regresión de Poisson. El 49,6\% de los hombres y el 54,7\% de las mujeres reportaron victimización; la agresión un $36,5 \%$ y $27,6 \%$, respectivamente. Se observaron prevalencias elevadas de víctima y de ofensor entre los participantes con actividades marginales de generación de ingresos y con uso de riesgo de alcohol. La victimización fue más probable en las mujeres que usaban vía parenteral y entre los hombres con consumo precoz de drogas ilegales, policonsumo de drogas ilegales y antecedentes penitenciarios. La agresión fue más probable entre los hombres receptores de tratamiento psicológico, consumo precoz de drogas ilegales, policonsumo de drogas ilegales y antecedentes penitenciarios, y entre las mujeres, aquellas que reportaron consumo precoz de drogas ilegales y que habían traficado. Se encontró una alta prevalencia de violencia en los usuarios de drogas ilícitas, especialmente entre aquellos involucrados en actividades de mercado y delincuencia. Los centros de tratamiento de drogas deberían considerar evaluar los antecedentes y los signos de violencia, y promover estrategias de salud comunitaria.

Palabras clave: Drogas ilegales; Violencia; Crimen; Género; Víctimaagresor.

\begin{abstract}
Illicit drug use is known to be associated with injuries resulting from violence. This study aims to estimate the prevalence of violence, for the last 12 months, in illicit drug users and study the victim-offender overlap, separately by sex. Persons using illicit drugs (502) were recruited in drug treatment facilities. Violence was assessed using four questions for victim and one for perpetrator in the last 12 months. Associations between violence and socio-demographic, substance use, crime and illegal drug market aspects were examined with Poisson regression models. Victimization was reported by $49.6 \%$ men and $54.7 \%$ women; offending by $36.5 \%$ and $27.6 \%$, respectively. Higher prevalence ratios of both victim and offender were observed among participants with marginal income generation activities and alcohol risk use. Victimization was more likely in women using parenteral route and among men with early illegal drug use, illegal polydrug use or history of imprisonment. Offending was more likely among men reporting psychological treatment, early illegal drug use, illegal polydrug use or past imprisonment, and women reporting early illegal drug use or trafficking. Thus, a high prevalence of violence (both victimization and perpetration) was found in illicit drug users, especially among those involved in market activities and crime. Drug treatment facilities should consider assessing for history and signs of violence and promote community health strategies.

Keywords: Illegal drug use; Violence; Crime; Gender; Victim-offender overlap.
\end{abstract}


$\mathrm{E}$ 1 abuso de drogas está asociado no solo con niveles altos de comorbilidad física y psiquiátrica y con la mortalidad, (Fridell y Nilson, 2004; Single, Robson, Rehm, y Xi, 1999; Torrens, Gilchrist, y Domingo-Salvany, 2011), sino también con una degradación progresiva de factores sociales, como las relaciones interpersonales y con el entorno, incluyendo la violencia (Boles y Miotto, 2003; Cunningham et al., 2003; MacDonald, Wells, Giesbrecht, y Cherpitel, 1999), como una consecuencia de la adicción a las drogas (UNODC, 1995; Wahler, 2012). Dichos factores contextuales pueden resultar del consumo en sí mismo o, a la inversa, pueden aumentar los riesgos del abuso de drogas, por tanto ejerciendo un efecto circular (Rutter, 2002).

Otros investigadores han examinado el contexto complejo interpersonal y social relacionado con el crimen y la violencia entre consumidores de drogas ilegales (Bennett, Holloway, y Farrington, 2008; Goldstein, 1985; White y Norman, 2000) y muchos estudios han destacado la importancia del contexto para entender los vínculos entre las drogas y la violencia (Arribas-Ibar, Suelves, Sanchez-Niubò, Domingo-Salvany, y Brugal, 2017; Caldentey et al., 2017; Reuter, 2009; Werb et al., 2011). Goldstein propuso un marco conceptual con tres modelos para explicar dicha relación: i) El Modelo Psicofarmacológico que resulta en cambios o deterioro cognitivos que precipitan la conducta delictiva, ii) El Modelo de Compulsividad Económica como vía para conseguir dinero para financiar el uso de drogas, y iii) El Modelo Sistémico inherente al sistema de distribución de drogas. Además, los tres modelos pueden solaparse (Goldstein, 1985).

Varios estudios que analizaron el modelo psicofarmacológico de Goldstein informaron que las propiedades químicas de las drogas ilegales pueden provocar conductas violentas (Cunningham et al., 2003; Pierce et al., 2015). Por ejemplo, Darke et al. encontró que era más probable que los usuarios habituales de metanfetaminas fuesen agresores en los últimos 12 meses, comparado con usuarios habituales de heroína, y propuso una posible relación entre las propiedades farmacológicas de la metanfetamina y el aumento relativo de crimen (Darke, Torok, Kaye, Ross, y McKetin, 2010). Otros estudios abordaron el segundo modelo de Goldstein, que sostiene que el uso de drogas puede contribuir a un número desproporcionado de eventos y crímenes violentos cometidos con fines económicos (Bennett et al., 2008; Klee y Morris, 1994; Topalli, Wright, y Fornango, 2002). Respecto del tercer modelo de Goldstein, varios estudios demostraron la influencia de las actividades relacionadas con las drogas ilegales (suministro y distribución) sobre la provocación de la violencia (Reuter, 2009; Ritter, 2006; Seddon, 2000). Un consenso creciente sostiene que gran parte de la violencia relacionada con las drogas tiene una naturaleza sistémica (Erickson, 2001). No obstante, los efectos sobre la violencia de la participación en el mercado de drogas y en otras actividades ilegales ha sido escasamente explorado, particularmente entre usuarios de drogas que buscan tratamiento en centros de salud.

$\mathrm{Al}$ investigar sobre conductas violentas, es importante tener en cuenta la correlación existente entre víctimas y agresores, el llamado "solapamiento víctima-agresor" (Jennings, Piquero, y Reingle, 2012). Cunningham et al. encontró que en urgencias, muchos de los pacientes con lesiones $(57,5 \%)$ dijeron ser víctima y agresor a la vez (Cunningham et al., 2003). De forma similar, otros estudios han encontrado que los agresores y las víctimas comparten un perfil sociodemográfico parecido y que, aparentemente, la etiología de la victimización y la agresión está relacionada (Daday, Broidy, Crandall, y Sklar, 2005).

Posick y Zimmerman sugieren que es necesario considerar las diferencias entre géneros para mejor comprender los perfiles de víctimas y agresores y el solapamiento entre los mismos (Posick y Zimmerman, 2015). Por tanto, nuestro estudio tiene como objetivo estimar la prevalencia de violencia entre usuarios de drogas ilegales que buscan tratamiento en centros de tratamiento de drogas en Cataluña (España), describir las características de víctimas y agresores, evaluar la asociación entre actividades del mercado ilegal y violencia, y estudiar el solapamiento víctima-agresor, para hombres y mujeres por separado.

Nuestra hipótesis era que sería más probable que los usuarios de drogas alguna vez implicados en tráfico de drogas ilegales $\mathrm{u}$ otras actividades ilegales reportarían haber sido víctimas y agresores durante el último año, y que dicha tendencia sería más notable en hombres que en mujeres. De manera similar, otra de nuestras hipótesis era que sería más probable que los sujetos que habían estado en prisión reportaran haber sido víctimas y agresores durante el último año, y que dicha tendencia sería la misma para hombre y mujeres. Por último, sospechábamos que no habría diferencias por género en el solapamiento víctima-agresor.

\section{Método}

\section{Entorno y población del estudio}

Este es un estudio transversal entre usuarios de drogas ilegales reclutados en centros de tratamiento y prevención en Cataluña (España) entre abril y junio del 2012. Seleccionamos centros del listado actualizado de centros públicos de tratamiento (2010) para abarcar todo el territorio, estratificados por tipo de centro. Con este fin, dividimos Cataluña en cinco áreas geográficas; las estrategias de muestreo fueron adaptadas para contemplar cada tipo de centro en una zona concreta. Priorizamos los Centros de Tratamiento Ambulatorio (CTA) por ser la puerta de entrada principal para recibir tratamiento en Cataluña. Finalmente, participaron 48 centros: 26 CTA, 12 Comunidades Terapéuticas residenciales profesionales (CTe), y 10 Centros de Reducción de Lesiones (CRL). Todos los 
CRL participaron en el estudio. El número de participantes a ser reclutados fue definido en base a la actividad y el tamaño de cada centro, aplicando sobremuestreo a los centros seleccionados más pequeños, en particular los CRL. Para los CRL, fijamos cuotas para género y país de nacimiento. Los participantes cumplimentaron un cuestionario con aspectos sociodemográficos, patrones de uso de sustancias, evaluación de servicios sanitarios, crimen, actividades de mercado y violencia. Este estudio fue aprobado por el Comité de Ética del IMIM (Instituto Hospital del Mar de Investigaciones Médicas). Los participantes firmaron un consentimiento informado antes del inicio del estudio. Los participantes de los CRL percibieron 10 euros como compensación. Un total de 558 usuarios de drogas ilegales fueron invitados a participar, 42 optaron por no participar y 2 no completaron el cuestionario, dejando 514 casos válidos. Los casos válidos tenían la siguiente distribución: 310 en CTA, 98 en CRL y 94 en CTe.

\section{Variables dependientes: víctima y agresor}

Valoramos violencia en base a cinco preguntas referidas a los últimos 12 meses, usando como referencia una guía publicada por la OMS sobre la implementación de encuestas comunitarias relacionadas con lesiones y violencia (Organización Mundial de la Salud, 2004). Las primeras cuatro preguntas aportaban información sobre violencia sufrida: 1) ¿Cuántas veces ha sido atacado/a, pateado/a, quemado/a o sufrido lesiones por arma de fuego, cuchillo, palo, o botella rota? 2) ¿Cuántas veces ha sido víctima de una agresión física que no implicó un arma?3) ¿Cuántas veces ha sido víctima de abuso sexual? y 4) ¿Cuántas veces ha sido víctima de abuso psicológico? Las respuestas fueron resumidas en una variable llamada "víctima" con el fin de obtener una valoración global de victimización de la muestra del estudio. Se consideró afirmativo cuando la persona encuestada informó haber sido víctima de cualquier tipo de violencia o agresión (física, con o sin arma, sexual o psicológica). Una pregunta hacía referencia a la violencia perpetrada: 5) ¿Cuántas veces ha agredido a otra persona físicamente? (con arma, dando una paliza, empujando, u de otra manera). En caso de reportar un episodio, el participante era considerado "agresor".

\section{Variables independientes}

Las variables independientes del estudio incluyeron aspectos sociodemográficos (país de nacimiento, ciudad, lugar de residencia, nivel de estudios, situación laboral, estado civil); tratamiento psicológico; patrones de uso de sustancias (edad de primer consumo de sustancia ilegal, administración parenteral, uso de riesgo de alcohol y policonsumo de drogas ilegales reciente), actividades relacionadas con el mercado de drogas ilegales (tráfico alguna vez y actividades de generación de ingresos) y crimen (prisión alguna vez).
Tratamiento psicológico se valoró para los 12 meses anteriores a la administración del cuestionario. Uso de riesgo de alcohol se midió con el Test de Identificación de los Trastornos Debidos al Consumo de Alcohol (AUDIT-C) respecto de los últimos 12 meses, considerando uso de riesgo de alcohol en hombres con una puntuación igual o superior a 4, y en mujeres con una puntuación igual o superior a 3 (Bradley et al., 2007). El policonsumo de drogas ilegales reciente implicó el uso diario de dos o más sustancias ilegales durante los últimos 30 días de consumo activo. Las actividades de generación de ingresos (AGI) diferenciaron entre actividades legales a cambio de dinero (de la familia, empleo legal, pensión o venta ambulante) y actividades ilegales y/o marginales (comercio sexual, robo, menudeo de drogas, mendigar, préstamo de traficante); cuando las respuestas incluyeron ambas categorías, se priorizó la clasificación como marginal.

\section{Análisis estadístico}

Los análisis se realizaron diferenciando entre género, de manera separada para violencia recibida (VÍCTIMA) y violencia perpetrada (AGRESOR). Las comparaciones, en los análisis descriptivos, fueron mediante los test de chi cuadrado y las pruebas t de Student. Para valorar el tamaño de efecto, se calculó la V de Cramer, aplicando los siguientes puntos de corte: pequeño 0,1 , mediano 0,3 y grande 0,5 , correspondiendo con las d de Cohen: 0,2, 0,5 y 0,8, respectivamente (Cohen, 1988). Calculamos la Razón de Prevalencia (RP) para identificar los factores asociados con violencia usando modelos de regresión de Poisson con varianza robusta. En estos modelos, usamos ecuaciones de estimación generalizadas (GEE) para tener en cuenta observaciones correlacionadas, según tipo de centro de reclutamiento (CRL, CTA o CTe). Todas las variables con valores $p \leq, 20$ en el análisis descriptivo se incluyeron en el modelo y después fueron omitidas paso a paso hasta que el modelo tuvo únicamente variables significativas $(p<, 05)$. Para cualquier variable que tuviese más de 15 respuestas sin contestar, creamos una categoría nueva para evitar perder dichos casos en el análisis. Las variables relacionadas con el uso de sustancias no se incluyeron en los modelos multifactoriales porque el consumo de drogas informado refería a periodos diferentes, dado que los participantes reclutados en distintos tipos de centros recibieron tratamiento en fechas distintas. Ajustamos un total de cuatro modelos, dos para víctima (hombres y mujeres) y dos para agresor, ajustados para edad y las variables sociodemográficas estadísticamente significativas (ver las notas al pie de página de cada tabla). Como último, para valorar el solapamiento víctima-agresor, el modelo resultante para víctima se ajustó por la condición de agresor, y el modelo resultante para agresor se ajustó por la condición de víctima. Todos los análisis se realizaron usando software SPSS, versión 18. 


\section{Resultados}

\section{Descripción de la muestra}

Entre los participantes válidos $(\mathrm{N}=514), 502$ informaron de violencia; 384 hombres $(76,5 \%)$ y 118 mujeres. La edad media era 37,9 (SD = 8,6) años. La mayoría de los participantes vino de CTA $(61,8 \%)$, mientras que los de CRL y CTe representaron aproximadamente $19 \%$ cada uno. La mayoría (91\%) había consumido cocaína o crack alguna vez en la vida, $86,5 \%$ cannabis, y $37,6 \%$ opioides (heroína y metadona). Una tercera parte $(34,2 \%)$ de los participantes tenía policonsumo de drogas ilegales. El $52,1 \%$ de los hombres y el $37,7 \%$ de las mujeres $(p<, 01)$ tuvo una edad de inicio en el uso de drogas ilegales menor de 14 años. En el último año, los hombres tenían un uso de riesgo más alto de alcohol que las mujeres (51,5\% y $39,8 \%$, respectivamente, $p<, 03)$. Más mujeres que hombres estaban en tratamiento psicológico $(38,9 \%$ y $25,8 \%$, respectivamente, $p<, 01)$. Otras variables referidas a los patrones de uso de drogas no mostraron diferencias por género. Más hombres que mujeres habían estado en prisión $(44,8 \%$ y $29,7 \%$, respectivamente, $p<, 01)$. Los hombres informaron de más actividades relacionadas con el tráfico de drogas, comparado con las mujeres $(52,9 \%$ y $42,4 \%$, respectivamente, $p<, 05)$. Por último, AGI ilegales y/o marginales fueron informadas con más frecuencia por hombres que por mujeres $(84,4 \%$ y $74,6 \%$, respectivamente, $p<, 02)$.

\section{Prevalencia de distintos tipos de violencia}

En los últimos 12 meses, la prevalencia de haber sido víctima fue $50,8 \%$ (49,6\% de los hombres y $54,7 \%$ de las mujeres) y de haber sido agresor fue $34,4 \%$ (36,5\% de los hombres y $27,6 \%$ de las mujeres) (Tabla 1). Aunque las mujeres con más frecuencia informaron ser víctimas y los hombres con más frecuencia informaron ser agresores, las diferencias no fueron estadísticamente significativas. Haber sido objeto de una agresión física (sin arma) o de abuso psicológico fue más común (aproximadamente 33\% cada uno) que haber sido objeto de una agresión con un arma $(17,4 \%)$ o de abuso sexual $(3,4 \%)$. Más hombres informaron haber sido víctimas de una agresión física (con y sin arma), mientras que una proporción más alta de mujeres informó haber sido objeto de abuso sexual y psicológico. Diferencias por género fueron significativas para todo tipo de violencia, exceptuando la agresión física sin arma.

\section{Análisis de víctima}

Las únicas variables sociodemográficas con una asociación significativa con "víctima" fueron situación laboral y residencia en hombres (Tabla 2). Para los hombres, todos los patrones de uso de drogas estaban asociados con la condición de víctima, mientras que, para las mujeres, las variables asociadas fueron uso de riesgo de alcohol, administración parenteral, y policonsumo de drogas ilegales. Es más, todas las variables de crimen y mercado fueron es-

Tabla 1. Tipos de violencia en usuarios de drogas ilegales en los últimos 12 meses para hombres y mujeres

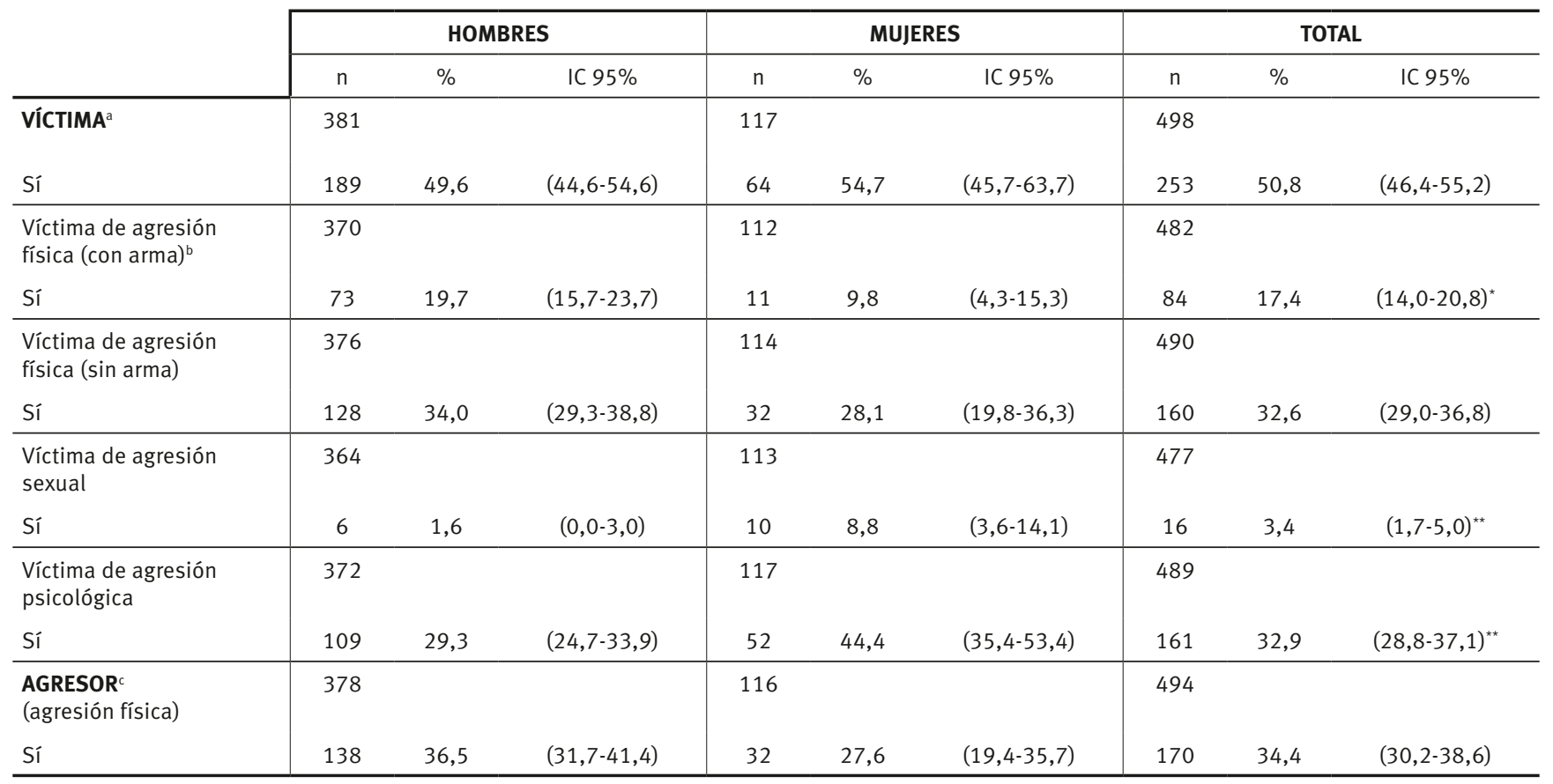

Nota. ${ }^{* *} p<0,01,{ }^{*} p<0,05$, Significación estadística en diferencias por género. a Incluyendo uno o más de los siguientes tipos de victimización: víctima de agresión física (con/sin arma), víctima de agresión sexual y abuso psicológico. ${ }^{b}$ Atacado/a, pateado/a, quemado/a o sufrido lesiones por arma de fuego, cuchillo, palo, botella rota, y otros. ' Agresión física. 
Tabla 2. Aspectos sociodemográficos de patrones de uso de sustancias y de crimen y mercado de drogas asociados con violencia sufrida (VÍCTIMA) en los últimos 12 meses, para hombres y mujeres

\begin{tabular}{|c|c|c|c|c|c|c|c|c|c|c|}
\hline & \multicolumn{5}{|c|}{ Hombres } & \multicolumn{5}{|c|}{ Mujeres } \\
\hline & \multirow{2}{*}{$\mathrm{N}$} & \multicolumn{4}{|c|}{ sí } & \multirow{2}{*}{$\mathrm{N}$} & \multicolumn{4}{|c|}{ sí } \\
\hline & & $\mathrm{n}$ & $\%$ & $p$ & V de Cramer & & $\mathrm{n}$ & $\%$ & $p$ & V de Cramer \\
\hline Total & 381 & 189 & 49,6 & & & 117 & 64 & 54,7 & & \\
\hline \multicolumn{11}{|l|}{ Edad } \\
\hline$\leq 35$ & 159 & 85 & 53,5 & \multirow{2}{*}{0,22} & \multirow{2}{*}{0,06} & 47 & 30 & 63,8 & \multirow{2}{*}{0,10} & \multirow{2}{*}{0,15} \\
\hline$\geq 36$ & 221 & 104 & 47,1 & & & 70 & 34 & 48,6 & & \\
\hline \multicolumn{11}{|l|}{ País de nacimiento } \\
\hline España & 341 & 164 & 48,1 & \multirow{2}{*}{0,09} & \multirow{2}{*}{0,09} & 108 & 59 & 54,6 & \multirow{2}{*}{0,96} & \multirow{2}{*}{0,01} \\
\hline Otros países ${ }^{\mathrm{a}}$ & 40 & 25 & 62,5 & & & 9 & 5 & 55,6 & & \\
\hline \multicolumn{11}{|l|}{ Ciudad } \\
\hline Menos de 100.000 habitantes; no AMB ${ }^{b}$ & 102 & 44 & 43,1 & \multirow{3}{*}{0,07} & \multirow{3}{*}{0,12} & 35 & 22 & 62,9 & & \\
\hline Más de 100.000 habitantes; no AMB & 84 & 37 & 44,0 & & & 17 & 7 & 41,3 & 0,33 & 0,14 \\
\hline Barcelona y $\mathrm{AMB}$ & 195 & 108 & 55,4 & & & 65 & 35 & 53,8 & & \\
\hline Nivel de estudios & & & & & & & & & & \\
\hline Bachillerato / Universidad & 112 & 47 & 42,0 & & & 41 & 18 & 43,9 & & \\
\hline Educaión secundaria & 156 & 84 & 53,8 & 0,14 & 0,10 & 42 & 24 & 57,1 & 0,18 & 0,17 \\
\hline Educación primaria/básica & 113 & 58 & 51,3 & & & 34 & 22 & 64,7 & & \\
\hline Situación laboral & & & & & & & & & & \\
\hline Empleado/a & 72 & 22 & 30,6 & & & 22 & 9 & 40,9 & & \\
\hline Desempleado/a/Ningún empleo anteriormente & 234 & 131 & 56,0 & $<0,01$ & 0,19 & 75 & 40 & 53,3 & 0,08 & 0,21 \\
\hline Discapacidad permanente/ pensionista & 74 & 36 & 48,6 & & & 20 & 15 & 75,0 & & \\
\hline Residencia & & & & & & & & & & \\
\hline Solo/a & 58 & 28 & 48,3 & & & 22 & 11 & 50,0 & & \\
\hline Casado/a o soltero/a con pareja & 63 & 29 & 46,0 & & & 29 & 17 & 58,6 & & \\
\hline Otros familiares/amigos & 136 & 48 & 35,3 & $<0,01$ & 0,34 & 46 & 25 & 54,3 & 0,78 & 0,12 \\
\hline En la calle/ ocupante ilegal & 56 & 49 & 87,5 & & & 8 & 6 & 75,0 & & \\
\hline Comunidad terapéutica & 62 & 31 & 50,0 & & & 10 & 5 & 50,0 & & \\
\hline Tratamiento psicológico ${ }^{c}$ & & & & & & & & & & \\
\hline No & 268 & 128 & 47,8 & 084 & 01 & 69 & 34 & 49,3 & 02 & 11 \\
\hline Sí & 94 & 46 & 48,9 & 0,04 & 0,01 & 43 & 26 & 60,5 & 0,23 & 0,11 \\
\hline PATRONES DE USO DE SUSTANCIAS PSICOACTIV & & & & & & & & & & \\
\hline Edad de inicio de consumo de alguna sustancia & & & & & & & & & & \\
\hline$\leq 14$ años & 198 & 116 & 58,6 & $<01$ & 018 & 43 & 26 & 60,5 & 034 & 0 \\
\hline$\geq 15$ años & 181 & 73 & 40,3 & 0,01 & 0,10 & 74 & 38 & 51,4 & 0,34 & 0,0 \\
\hline Uso de riesgo de alcohol $\mathrm{c,d}$ & & & & & & & & & & \\
\hline No & 186 & 82 & 44,1 & 004 & 011 & 71 & 33 & 46,5 & 003 & 021 \\
\hline Sí & 195 & 107 & 54,9 & & & 46 & 31 & 67,4 & & \\
\hline Administración parenteral alguna vez & & & & & & & & & & \\
\hline No & 207 & 91 & 44,0 & 01 & 013 & 73 & 34 & 46,6 & רח0 & 021 \\
\hline Sí & 172 & 98 & 57,0 & 0,01 & (3) & 44 & 30 & 68,2 & 0,02 & 0,21 \\
\hline Opioides alguna vez & & & & & & & & & & \\
\hline No & 149 & 57 & 38,3 & $<01$ & 014 & 55 & 26 & 47,3 & 013 & 001 \\
\hline Sí & 232 & 123 & 56,9 & 0,01 & 0,14 & 62 & 38 & 61,3 & (3) & 0,01 \\
\hline Cocaína y/o crack alguna vez & & & & & & & & & & \\
\hline No & 32 & 9 & 28,1 & 001 & 018 & 13 & 4 & 30,8 & 007 & 021 \\
\hline Sí & 346 & 178 & 51,4 & 0,01 & 0,10 & 104 & 60 & 57,7 & ו & 0,21 \\
\hline Policonsumo de drogas ilegales reciente & & & & & & & & & & \\
\hline No & 234 & 94 & 40,2 & $<0,02$ & 0,28 & 78 & 36 & 46,2 & 0,01 & 0,26 \\
\hline Sí & 126 & 87 & 69,0 & & & 35 & 26 & 74,3 & & \\
\hline
\end{tabular}




\begin{tabular}{|c|c|c|c|c|c|c|c|c|c|c|}
\hline Condena de prisión & & & & & & & & & & \\
\hline Nunca & 209 & 85 & 40,7 & \multirow{2}{*}{$<0,01$} & \multirow{2}{*}{0,19} & 82 & 41 & 50,0 & \multirow{2}{*}{0,12} & \multirow{2}{*}{0,14} \\
\hline Alguna vez & 170 & 102 & 60,0 & & & 35 & 23 & 65,7 & & \\
\hline \multicolumn{11}{|l|}{ Tráfico de drogas } \\
\hline Nunca & 180 & 79 & 43,9 & \multirow{2}{*}{$<0,05$} & \multirow{2}{*}{0,11} & 67 & 33 & 49,3 & \multirow{2}{*}{0,17} & \multirow{2}{*}{0,13} \\
\hline Alguna vez & 201 & 110 & 54,7 & & & 50 & 31 & 62,0 & & \\
\hline Familia/amigos/colegas & 28 & 8 & 28,6 & \multirow{3}{*}{0,05} & \multirow{3}{*}{0,13} & 13 & 6 & 46,2 & \multirow{3}{*}{0,07} & \multirow{3}{*}{0,21} \\
\hline Traficante/ fuentes marginales & 145 & 71 & 49,0 & & & 44 & 19 & 43,2 & & \\
\hline Ambos & 207 & 110 & 53,1 & & & 60 & 39 & 65,0 & & \\
\hline \multicolumn{11}{|c|}{ Actividades de generación de ingresos } \\
\hline Legal e & 59 & 18 & 30,5 & \multirow{2}{*}{0,01} & \multirow{2}{*}{0,16} & 30 & 11 & 36,7 & \multirow{2}{*}{0,02} & \multirow{2}{*}{0,21} \\
\hline Ilegal y/o marginal e & 322 & 171 & 53,1 & & & 87 & 53 & 60,9 & & \\
\hline
\end{tabular}

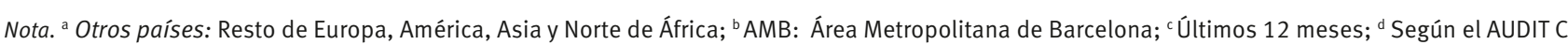
(Bradley et al., 2007); ' Actividades legales: dinero obtenido de la familia, pareja, empleo legal, pensión o venta ambulante; Actividades ilegales y/o marginales: dinero obtenido de comercio sexual, robo, menudeo de drogas, mendigar, o préstamo de traficante.

Tabla 3. Modelos de regresión de Poisson explorando factores asociados con la victimización en los últimos 12 meses para hombres y mujeres

\begin{tabular}{|c|c|c|c|c|c|c|}
\hline & \multicolumn{3}{|c|}{ Hombres $^{\text {a }}$} & \multicolumn{3}{|c|}{ Mujeres ${ }^{b}$} \\
\hline & N (376) & $\mathrm{RP}^{\mathrm{c}}$ & $95 \% I^{c}$ & $\mathrm{~N}(117)$ & $\mathrm{RP}$ & IC $95 \%$ \\
\hline \multicolumn{7}{|l|}{ Edad de inicio de consumo de alguna sustancia ilegal } \\
\hline$\geq 15$ años & 179 & 1 & & & & \\
\hline$\leq 14$ años & 197 & 1,3 & $(1,1-1,4)^{\star}$ & & & \\
\hline \multicolumn{7}{|l|}{ Uso de riesgo de alcohol ${ }^{\mathrm{d}}$} \\
\hline No & 184 & 1 & & 71 & 1 & \\
\hline Sí & 192 & 1,2 & $(1,1-1,4)^{\star}$ & 46 & 1,5 & $(1,3-1,7)^{\star}$ \\
\hline \multicolumn{7}{|l|}{ Administración parenteral alguna vez } \\
\hline No & & & & 73 & 1 & \\
\hline Sí & & & & 44 & 1,5 & $(1,1-1,7)^{\star}$ \\
\hline \multicolumn{7}{|l|}{ Policonsumo de drogas ilegales reciente } \\
\hline No & 232 & 1 & & & & \\
\hline Sí & 124 & 1,5 & $(1,1-2,0)^{\star}$ & & & \\
\hline Sin contestar ${ }^{e}$ & 20 & 1,0 & $(0,9-1,2)$ & & & \\
\hline \multicolumn{7}{|l|}{ Condena de prisión } \\
\hline Nunca & 208 & 1 & & & & \\
\hline Alguna vez & 168 & 1,3 & $(1,2-1,5)^{\star}$ & & & \\
\hline \multicolumn{7}{|l|}{ Actividades de generación de ingresos } \\
\hline Legal ${ }^{\mathrm{f}}$ & 59 & 1 & & 30 & 1 & \\
\hline llegal y/o marginal ${ }^{\mathrm{f}}$ & 317 & 1,3 & $(1,1-1,5)^{\star}$ & 87 & 1,4 & $(1,2-1,6)^{\star}$ \\
\hline
\end{tabular}

Nota. ${ }^{*} p<0,05 ;{ }^{a}$ Regresión de Poisson con varianza robusta, ajustado por edad y país de nacimiento; ${ }^{\text {b }}$ Regresión de Poisson con varianza robusta, ajustado

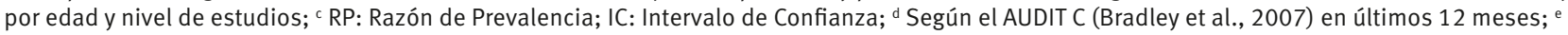

Creamos una categoría para variables sin contestar para evitar perder dichos casos del análisis; ${ }^{\mathrm{f}}$ Actividades legales: dinero obtenido de la familia, pareja, empleo legal, pensión o venta ambulante; Actividades ilegales y/o marginales: dinero obtenido de comercio sexual, robo, menudeo de drogas, mendigar, o préstamo de traficante.

tadísticamente significativas para hombres, mientras que para las mujeres únicamente AGI lo fue.

Tabla 3 muestra los resultados del análisis multifactorial. Entre los hombres, se observaron RP más altos para consumo temprano de drogas ilegales $(\leq 14$ años $)(\mathrm{RP}=$
$1,3)$, uso de riesgo de alcohol $(\mathrm{RP}=1,2)$, policonsumo de drogas ilegales reciente $(\mathrm{RP}=1,5)$, prisión alguna vez ( $\mathrm{RP}$ $=1,3)$ e involucración en AGI ilegales y/o marginales $(\mathrm{RP}=$ 1,3). Entre las mujeres, una RP más alta para victimización estaba asociada con uso de riesgo de alcohol $(\mathrm{RP}=1,5)$, 
Tabla 4. Aspectos sociodemográficos de patrones de uso de sustancias y de crimen y mercado de drogas asociados con agresores en los últimos 12 meses, para hombres y mujeres

\begin{tabular}{|c|c|c|c|c|c|c|c|c|c|c|}
\hline & \multicolumn{5}{|c|}{ Hombres } & \multicolumn{5}{|c|}{ Mujeres } \\
\hline & \multirow{2}{*}{$\mathrm{N}$} & \multicolumn{4}{|c|}{ sí } & \multirow{2}{*}{$\mathrm{N}$} & \multicolumn{4}{|c|}{ sí } \\
\hline & & $\mathrm{n}$ & $\%$ & $\mathrm{p}$ & V de Cramer & & $\mathrm{n}$ & $\%$ & $\mathrm{p}$ & V de Cramer \\
\hline Total & 378 & 138 & 36,5 & & & 116 & 32 & 27,6 & & \\
\hline \multicolumn{11}{|l|}{ Edad } \\
\hline$\leq 35$ & 160 & 69 & 43,1 & \multirow{2}{*}{0,02} & \multirow{2}{*}{0,12} & 47 & 18 & 38,3 & \multirow{2}{*}{0,03} & \multirow{2}{*}{0,20} \\
\hline$\geq 36$ & 217 & 69 & 31,8 & & & 69 & 14 & 20,3 & & \\
\hline \multicolumn{11}{|l|}{ País de nacimiento } \\
\hline España & 339 & 127 & 37,5 & \multirow{2}{*}{0,26} & \multirow{2}{*}{0,06} & 107 & 31 & 29,0 & \multirow{2}{*}{0,25} & \multirow{2}{*}{0,12} \\
\hline Otros países ${ }^{\mathrm{a}}$ & 39 & 11 & 28,2 & & & 9 & 1 & 11,1 & & \\
\hline \multicolumn{11}{|l|}{ Ciudad } \\
\hline Menos de 100.000 habitantes; no AMB & 101 & 33 & 32,7 & \multirow{3}{*}{0,19} & \multirow{3}{*}{0,09} & 35 & 11 & 31,4 & & \\
\hline Más de 100.000 habitantes; no AMB & 83 & 25 & 30,1 & & & 17 & 3 & 17,6 & 0,57 & 0,10 \\
\hline Barcelona y $\mathrm{AMB}$ & 191 & 77 & 40,3 & & & 63 & 17 & 27,0 & & \\
\hline Nivel de estudios & & & & & & & & & & \\
\hline Bachillerato / Universidad & 112 & 37 & 33,0 & & & 40 & 10 & 25,0 & & \\
\hline Educación secundaria & 153 & 63 & 41,2 & 0,30 & 0,08 & 43 & 11 & 25,6 & 0,68 & 0,08 \\
\hline Educación primaria/básica & 113 & 38 & 33,6 & & & 33 & 11 & 33,3 & & \\
\hline Situación laboral & & & & & & & & & & \\
\hline Empleado/a & 72 & 17 & 23,6 & & & 22 & 5 & 22,7 & & \\
\hline Desempleado/a/Ningún empleo anteriormente & 232 & 94 & 40,5 & 0,03 & 0,19 & 75 & 22 & 29,3 & 0,82 & 0,21 \\
\hline Discapacidad permanente/ pensionista & 73 & 26 & 35,6 & & & 19 & 5 & 26,3 & & \\
\hline Residencia & & & & & & & & & & \\
\hline Solo/a & 58 & 17 & 29,3 & & & 22 & 3 & 13,6 & & \\
\hline Casado/a o soltero/a con pareja & 62 & 21 & 33,9 & & & 29 & 7 & 24,1 & & \\
\hline Otros familiares/amigos & 135 & 37 & 27,4 & $<0,01$ & 0,23 & 45 & 15 & 33,3 & 0,31 & 0,20 \\
\hline En la calle/ ocupante ilegal & 56 & 33 & 58,9 & & & 7 & 2 & 28,6 & & \\
\hline Comunidad terapéutica & 61 & 26 & 42,6 & & & 11 & 5 & 45,5 & & \\
\hline Tratamiento psicológico ${ }^{c}$ & & & & & & & & & & \\
\hline No & 268 & 88 & 32,8 & 006 & 0 & 68 & 15 & 22,1 & 010 & 016 \\
\hline Sí & 91 & 40 & 44,0 & & & 44 & 16 & 36,4 & - & \\
\hline PATRONES DE USO DE SUSTANCIAS PSICOACTIV & & & & & & & & & & \\
\hline Edad de inicio de consumo de alguna sustancia & & & & & & & & & & \\
\hline$\leq 14$ años & 195 & 91 & 46,7 & 00 & ? ? & 44 & 17 & 38,6 & 0 & 10 \\
\hline$\geq 15$ años & 181 & 47 & 26,0 & 0,01 & 0,22 & 72 & 15 & 20,8 & 0,07 & 0,18 \\
\hline Uso de riesgo de alcohol c,d & & & & & & & & & & \\
\hline No & 184 & 43 & 23,4 & $<01$ & 0,7 & 69 & 9 & 13,0 & $<001$ & 039 \\
\hline Sí & 194 & 95 & 49,0 & 0,01 & 0 & 47 & 23 & 48,9 & 0,01 & ד, \\
\hline Administración parenteral alguna vez & & & & & & & & & & \\
\hline No & 208 & 66 & 31,7 & 003 & 012 & 73 & 17 & 23,3 & 017 & 013 \\
\hline Sí & 168 & 72 & 42,9 & ( & 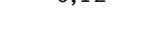 & 43 & 15 & 34,9 & 0,1 & 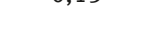 \\
\hline Opioides alguna vez & & & & & & & & & & \\
\hline No & 150 & 40 & 26,7 & $<001$ & 0.1 & 55 & 14 & 25,5 & 63 & 7 \\
\hline Sí & 228 & 98 & 43,0 & 0,01 & 0,2 & 61 & 18 & 29,5 & 0,0 & 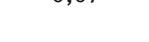 \\
\hline Cocaína y/o crack alguna vez & & & & & & & & & & \\
\hline No & 32 & 8 & 25,0 & (16 & 013 & 13 & 3 & 23,1 & 070 & 015 \\
\hline Sí & 344 & 129 & 37,5 & 0,10 & 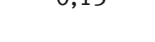 & 103 & 29 & 28,2 & 0,10 & 0,1 \\
\hline Policonsumo de drogas ilegales reciente & & & & & & & & & & \\
\hline No & 231 & 61 & 26,4 & $<0,1$ & 030 & 77 & 17 & 22,1 & 03 & 0.2 \\
\hline Sí & 126 & 71 & 56,3 & 0,01 & & 35 & 15 & 42,9 & 0,0 & \\
\hline
\end{tabular}




\begin{tabular}{|c|c|c|c|c|c|c|c|c|c|c|}
\hline CRIMEN Y MERCADO & & & & & & & & & & \\
\hline Condena de prisión & & & & & & & & & & \\
\hline Nunca & 209 & 61 & 29,2 & 0001 & 017 & 83 & 19 & 22,9 & 0707 & 017 \\
\hline Alguna vez & 167 & 76 & 45,5 & 8,01 & 0,17 & 33 & 13 & 39,4 & 0,07 & 0,17 \\
\hline Tráfico de drogas & & & & & & & & & & \\
\hline Nunca & 178 & 49 & 27,5 & 0001 & 018 & 67 & 13 & 19,4 & ר 0 & 21 \\
\hline Alguna vez & 200 & 89 & 44,5 & $\langle 0,01$ & 0,18 & 49 & 19 & 38,8 & 0,02 & 0,21 \\
\hline Proveedor/a de drogas & & & & & & & & & & \\
\hline Familia/amigos/colegas & 28 & 6 & 21,4 & & & 13 & 3 & 23,1 & & \\
\hline Traficante/ fuentes marginales & 145 & 53 & 36,6 & 0,20 & 0,09 & 43 & 9 & 20,9 & 0,35 & 0,13 \\
\hline Ambos & 204 & 79 & 38,7 & & & 60 & 20 & 33,3 & & \\
\hline Actividades de generación de in & & & & & & & & & & \\
\hline Legal $\mathrm{e}$ & 59 & 8 & 13,6 & $<001$ & 021 & 29 & 4 & 13,8 & 006 & 18 \\
\hline Ilegal y/o marginal $\mathrm{e}$ & 319 & 130 & 40,8 & $<0,01$ & 0,21 & 87 & 28 & 32,2 & 0,06 & 0,18 \\
\hline
\end{tabular}

Nota. a Otros países: Resto de Europa, América, Asia y Norte de África; ${ }^{\mathrm{D} A M B}$ : Área Metropolitana de Barcelona; ${ }^{c}$ Últimos 12 meses; ${ }^{\mathrm{d}}$ Según el AUDIT C (Bradley et al.,

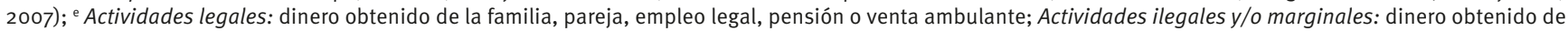
comercio sexual, robo, menudeo de drogas, mendigar, o préstamo de traficante.

Tabla 5. Modelos de regresión de Poisson explorando factores asociados con la perpetración de violencia (AGRESOR) los últimos 12 meses, para hombres y mujeres

\begin{tabular}{|c|c|c|c|c|c|c|}
\hline & \multicolumn{3}{|c|}{ Hombres $^{\mathrm{a}}$} & \multicolumn{3}{|c|}{ Mujeres $^{\mathrm{a}}$} \\
\hline & N (373) & $\mathrm{RP}^{\mathrm{b}}$ & IC $95 \%{ }^{b}$ & $\mathrm{~N}(116)$ & $\mathrm{RP}$ & IC 95\% \\
\hline \multicolumn{7}{|c|}{ Tratamiento psicológico ${ }^{c}$} \\
\hline No & 263 & 1 & & & & \\
\hline Sí & 91 & 1,4 & $(1,2-1,7)^{\star}$ & & & \\
\hline Sin contestar ${ }^{d}$ & 19 & 1,3 & $(1,1-1,5)$ & & & \\
\hline \multicolumn{7}{|c|}{ Edad de inicio de consumo de alguna sustancia ilegal } \\
\hline$\geq 15$ años & 179 & 1 & & 72 & 1 & \\
\hline$\leq 14$ años & 194 & 1,4 & $(1,3-1,6)^{\star}$ & 44 & 1,4 & $(1,1-1,8)^{*}$ \\
\hline \multicolumn{7}{|c|}{ Uso de riesgo de alcohol c,e } \\
\hline No & 182 & 1 & & 69 & 1 & \\
\hline Sí & 191 & 1,9 & $(1,8-2,1)^{\star}$ & 47 & 3,2 & $(1,9-5,3)^{\star}$ \\
\hline \multicolumn{7}{|c|}{ Policonsumo de drogas ilegales reciente } \\
\hline No & 229 & 1 & & & & \\
\hline Sí & 124 & 1,7 & $(1,5-1,9)^{\star}$ & & & \\
\hline Sin contestar ${ }^{d}$ & 20 & 1,2 & $(0,9-1,6)$ & & & \\
\hline \multicolumn{7}{|l|}{ Condena de prisión } \\
\hline Nunca & 208 & 1 & & & & \\
\hline Alguna vez & 165 & 1,4 & $(1,1-1,9)^{\star}$ & & & \\
\hline \multicolumn{7}{|l|}{ Tráfico de drogas } \\
\hline Nunca & & & & 67 & 1 & \\
\hline Alguna vez & & & & 49 & 1,5 & $(1,4-1,7)^{\star}$ \\
\hline \multicolumn{7}{|c|}{ Actividades de generación de ingresos } \\
\hline Legal $^{\mathrm{f}}$ & 59 & 1 & & 29 & 1 & \\
\hline Ilegal y/o marginal ${ }^{\mathrm{f}}$ & 314 & 2,0 & $(1,3-3,2)^{\star}$ & 87 & 2,1 & $(1,8-2,3)^{\star}$ \\
\hline
\end{tabular}

Nota. * $p$ < 0,05; a Regresión de Poisson con varianza robusta, ajustado por edad; b RP: Razón de Prevalencia; IC: Intervalo de Confianza; c Últimos 12 meses; ${ }^{d}$ Creamos una categoría para variables sin contestar para evitar perder dichos casos del análisis; ${ }^{e}$ Según el AUDIT C (Bradley et al., 2007); ${ }^{\mathrm{f}}$ Actividades legales: dinero obtenido de la familia, pareja, empleo legal, pensión o venta ambulante; Actividades ilegales y/o marginales: dinero obtenido de comercio sexual, robo, menudeo de drogas, mendigar, o préstamo de traficante. 
administración parenteral $(\mathrm{RP}=1,5)$ e involucración en AGI ilegales y/o marginales $(\mathrm{RP}=1,4)$.

\section{Análisis de agresor}

Adultos más jóvenes (edad $\leq 35$ años), hombres y mujeres, informaron con mayor frecuencia haber sido agresor (43,1\% de los hombres y $38,3 \%$ de las mujeres) que adultos de mayor edad. Otras variables sociodemográficas con una asociación significativa con "agresor" fueron situación laboral y residencia en hombres (Tabla 4). Para ambos géneros, la condición de agresor era significativamente más común entre aquellos con un inicio temprano en el uso de drogas (edad $\leq 14$ años) (46,7\% hombres y $38,6 \%$ mujeres), con un uso de riesgo de alcohol (49\% hombres y 48,9\% mujeres) o con consumo de drogas ilegales (56,3\% hombres y 42,9\% mujeres); además, era más probable que un hombre reportara la condición de agresor si usaba drogas por vía parenteral y si consumía opioides. La prevalencia de la condición de agresor era más alta entre los hombres que informaron implicación en algún delito (45,5\% de los que habían estado en prisión, 44,5\% de los que habían estado involucrados en tráfico de drogas, y 40,8\% de los que habían estado involucrados en AGI ilegales y/o marginales), mientras que era más probable que las mujeres que habían estado involucradas en tráfico de drogas reportasen la condición de agresor $(38,8 \%)$.

Tabla 5 muestra los resultados del análisis multifactorial para la condición de agresor. Era más probable que los hombres fuesen agresores si estaban en tratamiento psicológico $(\mathrm{RP}=1,4)$, habían tenido un inicio temprano en el uso de drogas ilegales (edad $\leq 14$ años) $(R P=1,4)$, con riesgo de alcohol $(\mathrm{RP}=1,9)$, policonsumo de drogas ilegales reciente $(\mathrm{RP}=1,7)$, habían estado en prisión ( $\mathrm{RP}$ $=1,4)$ e involucrados en AGI ilegales y/o marginales ( $\mathrm{RP}$ $=2,0$ ). Era más probable que las mujeres fuesen agresoras si habían tenido un inicio temprano en el uso de drogas ilegales (edad $\leq 14$ años) $(\mathrm{RP}=1,4)$, uso de riesgo de alcohol $(\mathrm{RP}=3,2)$, habían estado involucradas en el tráfico de drogas $(\mathrm{RP}=1,5)$ e involucradas en AGI ilegales $\mathrm{y} / \mathrm{o}$ marginales $(\mathrm{RP}=2,1)$.

\section{Solapamiento víctima-agresor}

De la muestra estudiada, 12 participantes contestaron únicamente a las preguntas para víctima o para agresor, pero no a ambas, resultando en 375 hombres y 115 mujeres para nuestro análisis del solapamiento víctima-agresor. Considerando a ambos géneros, 132 de las 245 víctimas también informó ser agresor $(53,9 \%)$; esta relación era diferente por género $(\mathrm{n}=107 / 183,58,5 \%$ hombres y $\mathrm{n}$ $=25 / 62,40,2 \%$ mujeres $)(p<, 02)$. De manera inversa, la gran mayoría de los que informaron ser agresor $(\mathrm{n}=166)$ también informó haber sido víctima ( $\mathrm{n}=132,79,5 \%)$ sin diferencias por género (hombres $\mathrm{n}=107 / 135,79,3 \%$ y mujeres $\mathrm{n}=25 / 31,80,6 \%)$.
Cuando se incluyó "agresor" en los modelos de víctimas (para cada género), el RP de ser víctima para un agresor masculino (en comparación con el hombre no agresor) fue $\mathrm{RP}=2,1$ (95\% IC: 2,0-2,3) y para una agresora $\mathrm{RP}=1,6$ (95\% IC: 1,4-1,8). En ambos casos, el uso de riesgo de alcohol perdió significancia para la asociación con ser víctima. Las demás variables fueron significativas, con la excepción de la administración parenteral para mujeres.

La RP de ser agresor para aquellos participantes que informaron ser víctimas era tres veces más elevada ( $\mathrm{RP}=3,0$; 95\% IC: 2,2-3,9) en hombres y dos veces más elevada ( $\mathrm{RP}=$ 2,3; $95 \%$ IC: 2,0-2,7) en mujeres, que aquellos que no informaron una historia de victimización. Las variables que perdieron significancia fueron haber estado en prisión para hombres y el uso temprano de drogas ilegales para mujeres.

\section{Discusión}

Observamos niveles altos de violencia reciente en los usuarios de drogas ilegales, hombres y mujeres, en tratamiento en centros de salud concretos; la mitad informó haber sido víctima (agresión física, abuso psicológico o sexual) y aproximadamente la tercera parte informó haber sido agresor. Aunque la prevalencia de la condición de víctima fue más alta para mujeres, y la condición de agresor fue más alta para hombres, dichas diferencias no fueron estadísticamente significativas. Para ambos géneros, las AGI ilegales y/o marginales tenían una asociación similar con la condición de víctima y de agresor, y al considerar el solapamiento víctima-agresor, el uso de riesgo de alcohol sólo estaba asociado con la condición de agresor. Además, por género, la victimización era más probable en hombres con un inicio temprano en el consumo de drogas ilegales, policonsumo de drogas ilegales e historia de prisión, mientras que era más probable en mujeres con una historia de administración parenteral. Respecto de la condición de agresor, era más común entre hombres que habían buscado tratamiento psicológico, los que informaron un inicio temprano en el consumo de drogas ilegales, policonsumo de drogas ilegales e historia de prisión, y era más común entre mujeres que informaron un inicio temprano en el consumo de drogas ilegales y que habían traficado.

Hubo diferencias por género en función de los tipos diferentes de violencia. La violencia psicológica y sexual fue más común entre las mujeres, mientras que la agresión física con un arma fue más común entre los hombres. En coherencia con estos hallazgos, un estudio europeo con 545 consumidores de drogas ilegales encontró que el 75,8\% de las mujeres y el 66,3\% de los hombres informó haber experimentado algún tipo de violencia. También en coherencia con nuestros resultados, una proporción más alta de mujeres informó haber sufrido violencia psicológica, comparado con consumidores de drogas masculinos (41,7\% y $30 \%$, respectivamente) (Stevens et al., 2007). En línea con otros 
estudios (Rodriguez y Griffin, 2005), encontramos que era más frecuente la involucración de hombres en conductas delictivas y en actividades relacionadas con el mercado de drogas ilegales, por lo que no es una sorpresa que la violencia con un arma sea más frecuente entre los hombres.

No obstante, debemos considerar algunas limitaciones. Primero, dado que los patrones de victimización son diferentes por género, y que un individuo puede haber sido objeto de varios tipos de violencia, todos los tipos de victimización fueron analizados conjuntamente para permitir un análisis con mayor robustez al aumentar el tamaño de la muestra; no obstante, esto no permitió el análisis de tipos de violencia específicos. Segundo, condiciones de victimización o de agresión previas a los 12 meses no fueron consideradas, permitiendo un mejor recuerdo y un análisis conjunto de otros eventos (tratamiento psicológico, policonsumo de drogas ilegales y uso de riesgo de alcohol) que ocurrieron durante el mismo periodo. Tercero, los autoinformes dependen de la memoria de los informantes, y también pueden ser influenciados por el sesgo de deseabilidad social. No obstante, algunos estudios con usuarios de drogas han mostrado la validez de los resultados transversales a pesar de haber sido autoinformados (Maisto, McKay, y Connors, 1990). En caso de informar sobre la violencia de manera insuficiente, las estimaciones de prevalencia también serían subestimadas. De todas maneras, para algunas formas concretas (e.g., abuso psicológico) no podemos descartar la posibilidad de que se haya informado con exageración, debido a las diferencias individuales en sensibilidad a la violencia (Collyer, Brell, Moster, y Furey, 2011). Cuarto, el reclutamiento se hizo en centros de salud, y, por tanto, los resultados pueden no ser generalizables a la población que no busca tratamiento. Por último, el diseño del estudio no permite realizar inferencias respecto de la causalidad de la violencia y las variables independientes.

Los niveles informados de violencia entre usuarios de drogas ilegales eran altos, en el mismo rango encontrado por estudios anteriores realizados con esta población, aunque varios tipos de victimización, pero solo agresión, física fueron considerados en este estudio. También respecto de los últimos 12 meses, Darke et al. encontraron que alrededor del $41 \%$ de los usuarios de metanfetaminas y de heroína habían cometido un delito violento, y que el $46 \%$ habían sido víctima de violencia (Darke et al., 2010) y otro estudio con usuarios de drogas ilegales (usuarios de metanfetaminas, cocaína y heroína) encontró que el $48 \%$ de los mismos había sufrido algún tipo de violencia física y el 34,5\% alguna agresión física en los últimos 6 meses (Martin et al., 2008). Un estudio en urgencias encontró que el 40,7\% de pacientes con lesiones que habían consumido alcohol o sustancias ilegales informaron haber sido víctimas de abuso sexual, violencia con arma, empujones, u otro tipo, y que el $35,6 \%$ fueron agresores (Cunningham et al., 2003), cifras que se acercan a las de nuestro estudio. La prevalencia de violencia informada por la población general es bastante menor, con cifras entre el 1,1\% para victimización violenta al menos una vez (Lauritsen y Rezey, 2013) hasta el 3,9\% en hombres y el $3 \%$ en mujeres para violencia general en los últimos 12 meses (Tjaden y Thoennes, 2000).

Interesantemente, nuestros hallazgos son coherentes con los modelos de Goldstein. El hecho de una edad de inicio temprana en el uso de drogas ilegales (antes de los 15 años) estaba asociado con la victimización y ser agresor en hombres, y la agresión en las mujeres puede considerarse relacionada con el primer modelo de Goldstein, que considera que el uso de drogas resulta en deterioro cognitivo (exacerbado con el paso de años de consumo de drogas) que puede desencadenar una conducta delictiva. Es más, varios estudios han mostrado como los efectos de algunas drogas, incluyendo anfetaminas, benzodiazepinas y crack/ cocaína, pueden causar conductas agresivas y violentas (Albertson, Walby y Derlet, 1995; Davis, 1996; Kuhns, 2005; Macdonald et al., 2003; Sommers, Baskin, y Baskin-Sommers, 2006). En nuestro estudio, un consumo elevado de alcohol estaba asociado con la condición de agresor, en hombres y mujeres.

Según el segundo modelo (compulsividad económica), encontramos que era más probable que los hombres que habían estado en prisión fuesen tanto víctima como agresor, lo que sugiere que habían participado en violencia y/o delitos económicos con el fin de sostener su uso costoso de drogas. Otros estudios, aunque no recientes, han aportado evidencia que apoya esta hipótesis: usuarios de heroína informaron haber cometido crímenes violentos después de haber sido usuarios de drogas (Parker y Newcombe, 1987), como también fue el caso de usuarios de cocaína crack (Parker y Bottomley, 1996).

Respecto del tercer modelo, la involucración en AGI ilegales y/o marginales estaba asociada con ser víctima y/o agresor para ambos géneros, y de manera específica, era más probable que las mujeres involucradas en actividades relacionadas con el mercado de drogas ilegales fuesen agresoras. La evidencia muestra que la necesidad de obtener dinero para comprar drogas en contextos sociales desfavorables induce a muchos usuarios a participar en actividades ilegales y/o marginales (Carpentier, 2007; Kuhns, 2005; Richardson et al., 2015). De forma similar, en un estudio sobre mujeres que ejercen el comercio sexual (de las cuales gran mayoría también usaban drogas), Gilchrist et al. destacaron que las participantes informaron haber sido objeto, con frecuencia, de la agresión física violenta (47\%) y la agresión sexual (39\%) mientras trabajaban (Gilchrist et al., 2001). Mientras que la involucración en el mercado de la droga es más elevada en los hombres (Anderson, 2001) y pueda estar relacionada con un consumo más alto de drogas (Office of Applied Studies, 1997), en nuestro estudio solo era más probable que las mujeres involucradas en el tráfico de drogas fuesen agresoras y no víctimas. 
El solapamiento víctima-agresor fue muy alto para ambos géneros, como habían encontrado Darke et al. (Darke et al., 2010). De manera destacada, el solapamiento fue más alto para agresores de ambos géneros, que también reportaron ser víctimas; la proporción de víctimas que informó ser, a la vez, agresores físicos, fue más baja y con diferencias por género (más alta en hombres). Este hallazgo no es sorprendente, dado el hecho que la condición de "agresor" refiere únicamente a la violencia física, mientras que las víctimas podrían serlo también por violencia sexual o psicológica, y la violencia informada por mujeres víctimas solía ser de naturaleza sexual y psicológica. Cuando valoramos únicamente la violencia física (datos no mostrados), no hubo diferencias de género en la probabilidad que una víctima se convirtiese en agresor, y vice versa, como se ha informado en la población general (Shäffer, 2004). Otro punto importante a destacar es el papel confusor del uso de riesgo de alcohol y su asociación con la condición de víctima en los modelos de solapamiento. En nuestro estudio, el uso de riesgo de alcohol estaba asociado con ambas condiciones de agresor y víctima; no obstante, cuando ajustamos el modelo de víctima con la variable "agresor", desapareció la asociación con el alcohol. Aunque algunos estudios que han valorado únicamente la relación entre el uso de alcohol y la condición de víctima hallaron asociaciones entre ambos (Giancola, 2015; Strunin et al., 2015; Testa y Hoffman, 2012), nuestros resultados son coherentes con otros estudios que mencionan al alcohol como la sustancia más frecuentemente relacionada con conductas agresivas y violentas (Bushman y Cooper, 1990; Crane, Godleski, Przybyla, Schlauch, y Testa, 2016; Testa y Derrick, 2014).

Encontramos una prevalencia elevada de violencia, tanto como víctima y como agresor, entre usuarios de drogas ilegales, especialmente entre aquellos implicados en crímenes y actividades relacionadas con el mercado de drogas ilegales. También hubo una asociación entre el uso de riesgo de alcohol y la violencia, en particular con la condición de agresor. Respecto de esta prevalencia elevada de violencia informada por parte de usuarios de drogas ilegales, los centros de tratamiento de drogas deberían valorar signos de violencia y fomentar el desarrollo de programas de prevención y tratamiento para abordar la violencia en ambos géneros de forma similar. Concretamente, el uso de herramientas de cribado podría ser útil con el fin de detectar cualquier tipo de violencia entre usuarios de drogas ilegales, y sería recomendable implementar intervenciones basadas en la evidencia para abordar la victimización y la perpetración desde una perspectiva de género. Estudios futuros podrían valorar la efectividad de estas estrategias.

\section{Reconocimientos}

El Gobierno de España concedió una ayuda a este estudio: Instituto de Salud Carlos III -FIS PI11/01358. La
Agencia de Gestión de Ayudas Universitarias y de Investigación (AGAUR 2009 SGR 718) y FIS-Redes de investigación cooperativa RD12/0028/0018 también concedieron ayudas financieras. El proyecto europeo JUST/2010/DPIP/ AG/1410 también contribuyó a la implementación de la encuesta. Las ayudas concedidas por FIS, AGAUR y EUJUST no asumieron papel alguno en el diseño del estudio o en la recolección, análisis e interpretación de los datos, en la redacción del manuscrito, o en cualquier decisión relacionada con la presentación del manuscrito para su publicación.

Agradecemos a los participantes, los centros colaboradores y sus profesionales. También agradecemos a Dave Macfarlane por editar y revisar el texto en inglés.

\section{Conflicto de intereses}

Los autores declaran la inexistencia de conflictos de intereses en relación al estudio, su autoría, y/o la publicación de este manuscrito.

\section{Referencias}

Albertson, T. E., Walby, W. F. y Derlet, R. W. (1995). Stimulant-induced pulmonary toxicity. Chest, 108, 1140-1149. doi:10.1378/chest.108.4.1140.

Anderson, T. L. (2001). Drug Use and Gender. In Faupel, C. E. y Roman, P. M. (Eds.), Encyclopedia of Criminology and Deviant Behavior, Vol. IV: Self-Destructive Behavior and Disvalued Identity (pp. 286-289). Philadelphia, PA: Taylor \& Francis.

Arribas-Ibar, E., Suelves, J. M., Sanchez-Niubò, A., Domingo-Salvany, A. y Brugal, T. M. (2017). Suicidal behaviours in male and female users of illicit drugs recruited in drug treatment facilities. Gaceta Sanitaria, 31(4), 292298. doi:10.1016/j.gaceta.2016.11.011.

Bennett, T., Holloway. K. y Farrington, D. (2008). The statistical association between drug misuse and crime: A meta-analysis. Aggression and Violent Behavior, 13, $107-$ 118. doi:10.1016/j.avb.2008.02.001.

Boles, S. M. y Miotto, K. (2003). Substance abuse and violence. Aggression and Violent Behavior, 8, 155-174. doi:10.1016/S1359-1789(01)00057-X.

Bradley, K. A., DeBenedetti, A. F., Volk, R. J., Williams, E. C., Frank, D. y Kivlahan, D. R. (2007). AUDIT-C as a brief screen for alcohol misuse in primary care. Alcoholism: Clinical and Experimental Research, 31, 1208-1217. doi:10.1111/j.1530-0277.2007.00403.x.

Bushman, B. J. y Cooper, H. M. (1990). Effects of alcohol on human aggression: an integrative research review. Psychological Bulletin, 107, 341-354. doi: 10.1037/00332909.107.3.341.

Caldentey, C., Tirado Muñoz, J., Ferrer, T., Fonseca Casals, F., Rossi, P., Mestre-Pintó, J. I. y Torrens Melich, M. (2017). 
Intimate partner violence among female drug users admitted to the general hospital: screening and prevalence. Adicciones, 29, 172-179. doi:10.20882/adicciones.738.

Carpentier, C. (2007). Drugs in focus: Drugs and crime- a complex relationship towards a definition of drug-related cri$m e$. Lisbon: European Monitoring Centre for Drugs and Drug Addiction.

Cohen, J. (1988). Statistical power analysis for the behavioral sciences. (2nd ed.). Hillsdale, NJ: Lawrence Erlbaum.

Collyer, C. E., Brell, A., Moster, A. y Furey, J. (2011). Individual differences in sensitivity to violence. Perceptual and Motor Skills, 113, 703-714. doi:10.2466/07.17.21. PMS.113.6.703-714.

Crane, C. A., Godleski, S. A., Przybyla, S. M., Schlauch, R. C. y Testa, M. (2016). The Proximal Effects of Acute Alcohol Consumption on Male-to-Female Aggression: A Meta-Analytic Review of the Experimental Literature. Trauma Violence Abuse, 17, 520-531. doi:10.1177/1524838015584374.

Cunningham, R., Walton, M. A., Maio, R. F., Blow, F. C., Weber, J. E. y Mirel, L. (2003). Violence and substance use among an injured emergency department population. Academic Emergency Medicine, 10, 764-775. doi:10.1197/ aemj.10.7.764.

Daday, J. K., Broidy, L. M., Crandall, C. S. y Sklar, D. P. (2005). Individual, Neighborhood, and Situational Factors Associated with Violent Victimization and Offending. Criminal Justice Studies, 18, 215-235. doi:10.1080/14786010500287347.

Darke, S., Torok, M., Kaye, S., Ross, J. y McKetin, R. (2010). Comparative rates of violent crime among regular methamphetamine and opioid users: Offending and victimization. Addiction, 105, 916-91. doi:10.1111/j.13600443.2009.02872.x.

Davis, W. M. (1996). Psychopharmacologic violence associated with cocaine abuse: kindling of a límbic dyscontrol syndrome?. Neuro-Psychopharmacology and Biological Psychiatry, 20, 1273-1300. doi:10.1111/j.13600443.2009.02872.x.

Erickson, P. G. (2001). Drugs, violence, and public health: What does a harm reduction approach have to offer? Sensible solutions to the urban drug problem. In Basham P. (Ed). Canada, Vanouver: Fraser Institute Digital Publication.

Fridell, M. y Nilson, M. (2004). Co-morbidity-drug use and mental disorders. Lisbon: European Monitoring Centre for Drugs and Drug Addiction.

Giancola, P. R. (2015). Development and Evaluation of Theories of Alcohol-Related Violence: Covering a 40Year Span. Substance Use Misuse, 50, 1182-1187. doi:10.31 09/10826084.2015.1010836.

Gilchrist, G., Taylor, A., Goldberg, D., Mackie, C., Denovan, A. y Green, S. T. (2001). Behavioural and Lifestyle Study of Women Using a Drop-In Centre for Fema- le Street Sex Workers in Glasgow, Scotland: A 10 Year Comparative Study. Addiction Research E Theory, 9, 43-58. doi:10.3109/16066350109141771.

Goldstein, P. J. (1985). The Drugs/Violence Nexus: A Tripartite Conceptual Framework. Journal of Drug Issues, 39, 143-174. doi:10.1177/002204268501500406.

Jennings, W. G., Piquero, A. R. y Reingle, J. M. (2012). On the overlap between victimization and offending: A review of the literature. Aggression and Violent Behavior, 17, 16-26. doi:10.1016/j.avb.2011.09.003.

Klee, H. y Morris, J. (1994). Crime and drug misuse: economic and psychological aspects of the criminal activites of heroin and amphetamine injectors. Addiction Research, 1, 377-386. doi:10.3109/16066359409005204.

Kuhns, J. B. (2005). The dynamic nature of the drug use/serious violence relationship: a multi-causal approach. Violence Victim, 20, 433-454. doi:10.1891/vivi.2005.20.4.433.

Lauritsen, J. L. y Rezey, M. L. (2013). Measuring the prevalence of crime with the national crime victimization survey. Washington, DC: U.S. Bureau of Justice Statistics.

MacDonald, S., Anglin-Bodrug, K., Mann, R. E., Erickson, P., Hathaway, A., Chipman, M. y Rylett, M. (2003). Injury risk associated with cannabis and cocaine use. A review. Drug and Alcohol Dependence, 72, 99-115. doi:10.1016/ S0376-8716(03)00202-3.

MacDonald, S., Wells, S., Giesbrecht, N. y Cherpitel, C. J. (1999). Demographic and substance use factors related to violent and accidental injuries: Results from an emergency room study. Drug and Alcohol Dependence, 55, 53-61. doi:10.1016/S0376-8716(98)00184-7.

Maisto, S. A., McKay, J. R. y Connors, G. J. (1990). Self-report issues in substance abuse: State of the art and future directions. Behavioral Assessment, 12, 117-134.

Martin, I., Palepu, A., Wood, E., Li, K., Montaner, J. y Kerr, T. (2008). Violence among street-involved youth: The role of methamphetamine. European Addiction Research, 15, 32-8. doi:10.1159/000173007.

Office of Applied Studies. (1997). 1996 National Household Server. Washington, DC: Substance Abuse and Mental Health Services Association.

Organización Mundial de la Salud. (2004). Guidelines for Conducting Community Surveys on Injuries and Violence. Geneva: Author.

Parker, H. y Bottomley, T. (1996). Crack cocaine and drugs: crime careers. London: Home Office Publications Unit.

Parker, H. y Newcombe, R. (1987). Heroin use and acquisitive crime in an English community. British Journal of Sociology, 38, 331-350. doi:10.2307/590692.

Pierce, M., Hayhurst, K., Bird, S. M., Hickman, M., Seddon, T., Dunn, G. y Millar, T. (2015). Quantifying crime associated with drug use among a large cohort of sanctioned offenders in England and Wales. Drug and Alcohol Dependence, 155, 52-59. doi:10.1016/j.drugalcdep.2015.08.018. 
Posick, C. y Zimmerman, G. M. (2015). Person-in-context: insights on contextual variation in the victim-offender overlap across schools. Journal of Interpersonal Violence, 30, 1432-1455. doi:10.1177/0886260514540327.

Reuter, P. (2009). Systemic violence in drug markets. Crime, Law and Social Change, 52, 275-284. doi:10.1007/s10611009-9197-x.

Richardson, L. A., Long, C., DeBeck, K., Nguyen, P., Milloy, M. J., Wood, E. y Kerr, T. H. (2015). Socioeconomic marginalisation in the structural production of vulnerability to violence among people who use illicit drugs. Journal of Epidemiology Community Health, 69, 686-692. doi:10.1136/jech-2014-205079.

Ritter, A. (2006). Studying illicit drug markets: Disciplinary contributions. International Journal of Drug Policy, 17 , 453-463. doi:10.1016/j.drugpo.2006.09.004.

Rodriguez, N. y Griffin, M. (2005). Gender Differences in Drug Market Activities: A Comparative Assessment of Men and Women's Participation in the Drug Market. Washington, DC: National Institute of Justice.

Rutter, M. (2002). The interplay of nature, nurture, and developmental influences: the challenge ahead for mental health. Archives of General Psychiatry, 59, 9961000. doi:10.1001/archpsyc.59.11.996.

Seddon, T. (2000). Explaining the drug-crime link: Theoretical, policy and research issues. Journal of Social Policy, 29, 95-107. doi:10.1017/S0047279400005833.

Shäffer, J. N. (2004). The Victim-Offender Overlap: Specifying the Role of Peer Groups (Doctoral thesis, Pennsylvania State University). Recuperado de https://www.ncjrs.gov/ pdffiles1/nij/grants/205126.pdf.

Single, E., Robson, L., Rehm, J. y Xi, X. (1999). Morbidity and mortality attributable to alcohol, tobacco, and illicit drug use in Canada. American Journal of Public Health, 89, 385-390. doi:10.2105/AJPH.89.3.385.

Sommers, I., Baskin, D. y Baskin-Sommers, A (2006). Methamphetamine use among young adults: health and social consequences. Addictive Behaviors, 31, 1469-1476. doi: 10.1016/j.addbeh.2005.10.004.

Stevens, A., Berto, D., Frick, U., Kerschl, V., McSweeney, T., Schaaf, S.,... Werdenich, W. (2007). The Victimization of Dependent Drug Users. European Journal of Criminology, 4, 385-408. doi:10.1177/1477370807080719.

Strunin, L., Díaz-Martínez, L. R., Díaz-Martínez, A., Heeren, T., Winter, M., Kuranz, S.,... Solís-Torres, C. (2015). Drinking Patterns and Victimization among Male and Female Students in Mexico. Alcohol and Alcoholism, 50, 226-235. doi:10.1093/alcalc/agu092.

Testa, M. y Derrick, J. L. (2014). A daily process examination of the temporal association between alcohol use and verbal and physical aggression in community couples. Psychology of Addictive Behaviors, 28, 127-138. doi:10.1037/a0032988.
Testa, M. y Hoffman, J. H. (2012). Naturally Occurring Changes in Women's Drinking From High School to College and Implications for Sexual Victimization. Journal of Studies on Alcohol and Drugs, 73, 26-33. doi:10.15288/ jsad.2012.73.26.

Tjaden, P. y Thoennes, N. (2000). Full Report of the Prevalence, Incidence, and consequences of violence against women. Washington, DC: National Institute of Justice.

Topalli, V., Wright, R. y Fornango, R. (2002). Drug dealers, robbery and retaliation: Vulnerability, Deterrence and the Contagion of Violence. British Journal of Criminoly, 42, 337-351. doi:10.1093/bjc/42.2.337.

Torrens, M., Gilchrist, G. y Domingo-Salvany, A. (2011). Psychiatric comorbidity in illicit drug users: Substance-induced versus independent disorders. Drug and Alcohol Dependence, 113, 147-156. doi:10.1016/j.drugalcdep.2010.07.013.

United Nations Office on Drugs and Crime. (1995). The social impact of drug abuse. Vienna: Author.

Wahler, E. A. (2012). The relationship of social stress, economic hardship, and psychological distress to addiction severity among kentucky substance abuse treatment participants (Doctoral thesis, University of Kentucky). Recuperado de https:/ / uknowledge.uky.edu/cgi/viewcontent.cgi? referer=https: / / www.google.com / \&httpsredir $=1$ \&article $=1000 \&$ context=csw_etds.

Werb, D., Rowell, G., Guyatt, G., Kerr, T., Montaner, J. y Wood, E. (2011). Effect of drug law enforcement on drug market violence: A systematic review. International Journal of Drug Policy, 22, 87-94. doi:10.1016/j.drugpo.2011.02.002.

White, H. y Norman, D. (2000). Dynamics of the Drug-Crime Relationship In Crimal Justice 2000. The nature of crime: continuity and change. Washington, DC: Bureau of Justice Statistics. 